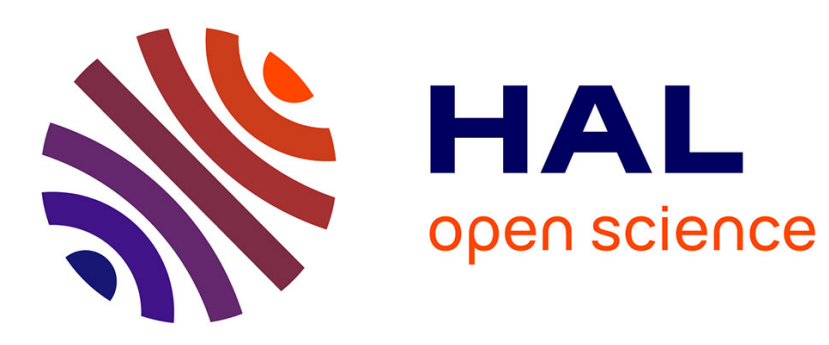

\title{
Strongly Correlated Charge Transport in Silicon Metal-Oxide-Semiconductor Field-Effect Transistor Quantum Dots
}

M. Seo, P. Roulleau, P. Roche, D. c. Glattli, M. Sanquer, X. Jehl, L. Hutin, S. Barraud, F. D. Parmentier

\section{To cite this version:}

M. Seo, P. Roulleau, P. Roche, D. c. Glattli, M. Sanquer, et al.. Strongly Correlated Charge Transport in Silicon Metal-Oxide-Semiconductor Field-Effect Transistor Quantum Dots. Physical Review Letters, 2018, 121, pp.027701. 10.1103/PhysRevLett.121.027701 . hal-01872901

\section{HAL Id: hal-01872901 https://hal.science/hal-01872901}

Submitted on 12 Sep 2018

HAL is a multi-disciplinary open access archive for the deposit and dissemination of scientific research documents, whether they are published or not. The documents may come from teaching and research institutions in France or abroad, or from public or private research centers.
L'archive ouverte pluridisciplinaire HAL, est destinée au dépôt et à la diffusion de documents scientifiques de niveau recherche, publiés ou non, émanant des établissements d'enseignement et de recherche français ou étrangers, des laboratoires publics ou privés. 


\title{
Strongly Correlated Charge Transport in Silicon Metal-Oxide-Semiconductor Field-Effect Transistor Quantum Dots
}

\author{
M. Seo, ${ }^{1}$ P. Roulleau, ${ }^{1}$ P. Roche, ${ }^{1}$ D. C. Glattli, ${ }^{1}$ M. Sanquer, ${ }^{2}$ X. Jehl, ${ }^{2}$ L. Hutin, ${ }^{3}$ S. Barraud,${ }^{3}$ and F. D. Parmentier ${ }^{1, *}$ \\ ${ }^{1}$ SPEC, CEA, CNRS, Université Paris-Saclay, CEA Saclay, 91191 Gif-sur-Yvette cedex, France \\ ${ }^{2}$ Université Grenoble Alpes, CEA, INAC-PHELIQS, 38000 Grenoble, France \\ ${ }^{3}$ CEA, LETI, Minatec Campus, 38000 Grenoble, France
}

(Received 22 May 2018; published 11 July 2018)

\begin{abstract}
Quantum shot noise probes the dynamics of charge transfers through a quantum conductor, reflecting whether quasiparticles flow across the conductor in a steady stream, or in syncopated bursts. We have performed high-sensitivity shot noise measurements in a quantum dot obtained in a silicon metal-oxidesemiconductor field-effect transistor. The quality of our device allows us to precisely associate the different transport regimes and their statistics with the internal state of the quantum dot. In particular, we report on large current fluctuations in the inelastic cotunneling regime, corresponding to different highly correlated, non-Markovian charge transfer processes. We have also observed unusually large current fluctuations at low energy in the elastic cotunneling regime, the origin of which remains to be fully investigated.
\end{abstract}

DOI: 10.1103/PhysRevLett.121.027701

Current fluctuations in a mesocopic conductor, or quantum shot noise [1], reflect the granularity of charge transfers across the conductor. By measuring low frequency current fluctuations, one can probe the correlations between subsequent charge transfers and quantify how random these transfers are. Those correlations are underpinned by the interplay between the quantum statistics of the particles flowing across a given conductor, electronic interactions, and the physical mechanisms giving rise to transport in the conductor. Rare, uncorrelated charge transfers lead to Poissonian shot noise $S_{I I}=2 e\langle I\rangle$, with $e$ the charge of the quasiparticles and $\langle I\rangle$ the average value of the dc current flowing across the conductor. Correlations between subsequent transfers are encoded in the Fano factor $F$, defined as the ratio between the shot noise and its Poissonian value. Fermionic statistics tend to impose some order on charge transport [2,3], characterized by reduced fluctuations $(F<1)$, or, in the case of perfectly ballistic conductors, fully noiseless transport $(F=0)$. While Coulomb interactions tend to do the same [4], they can, in some remarkable cases, give rise to positively correlated transport processes with super-Poissonian $(F>1)$ fluctuations, where charges flow in bursts through the conductor.

Coulomb-blockaded quantum dots are very rich systems, as they can not only present Poissonian and sub-Poissonian transport regimes [1,4], but also, depending on their internal structure, strongly correlated transport with super-Poissonian current fluctuations [5-17]. The latter regime corresponds to non-Markovian transport processes where the transfer of a charge across the dot changes its state, thereby influencing the next transfer event [5]. As a result, the quantum dot randomly switches between highly and poorly conducting channels while other parameters (e.g., temperature, bias voltage) remain fixed. This can happen in the sequential tunneling regime if several levels of a dot, with markedly different couplings to the leads, participate to transport. In that case the current across the quantum dot shows random telegraph signal features [15], yielding strongly enhanced fluctuations [6,13-15,17].

Inelastic cotunneling [18] is also expected to enhance the current fluctuations, as it is, by definition, a charge transfer process that changes the state of the quantum dot [5]. Several mechanisms leading to super-Poissonian shot noise in the inelastic cotunneling regime have been proposed, depending on the internal structure of the quantum dot and the respective chemical potentials of the dot and the leads. Some of these mechanisms are depicted in Fig. 1, for a $N$-charge quantum dot with a single excited state labeled $N^{\star}$. Inelastic cotunneling (blue arrows) events leave the dot in the excited state $N^{\star}$. In (1) (2), the $N-1 \leftrightarrow N^{\star}$ $\left(N^{\star} \leftrightarrow N+1\right)$ transition involving $N^{\star}$ sits in the bias window, allowing direct transport through the dot after the cotunneling event $[19,20]$. This process, usually referred to as cotunneling-assisted tunneling (CAST [10,21], or COSET $[12,17,22,23])$, is depicted as dashed red arrows in Fig. 1. It leads to enhanced fluctuations, as the system randomly switches between the blocked state $N$ and the conducting (excited) state $N^{\star}[7,9,10,12]$. In (3), all transitions are outside the bias window, and the dot is always in a blocked state. This can nonetheless lead to enhanced fluctuations: indeed, whether the quantum dot is blocked in the ground or excited state, elastic cotunneling (green dashed arrows in Fig. 1) with a priori different rates can occur. Inelastic cotunneling events will then randomly switch the quantum dot between states with different (albeit small) conductances, yielding super-Poissonian 


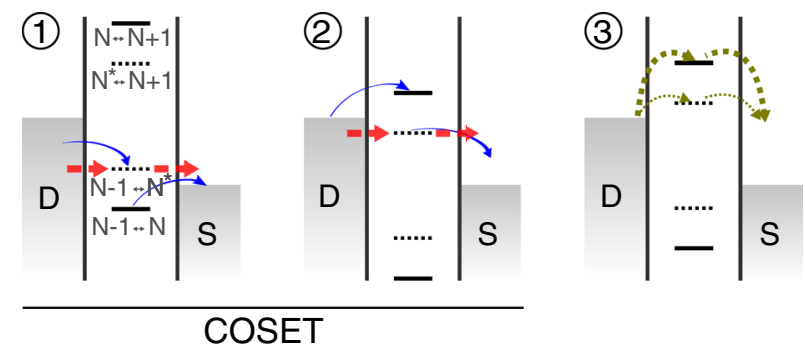

FIG. 1. Sketches of cotunneling processes giving rise to superPoissonian noise, described in the text. The thin blue arrows correspond to usual inelastic cotunneling; the thick, dashed, red arrows to COSET. In (3), the dashed green arrows correspond to elastic cotunneling with different rates depending on the state of the dot.

fluctuations $[10,12]$. This latter regime occurs if $\Delta^{*}<E_{c} / 3$, where $\Delta^{*}$ is the excited state energy and $E_{c}$ the charging energy $[10,12,17,19,21,23]$. Previous experimental works reported the observation of super-Poissonian noise in carbon nanotubes $[8,17]$ and in GaAs/AlGaAs [16] quantum dots in the inelastic cotunneling regime. In Refs. $[16,17]$, the structure of the excited states suggest that the enhanced shot noise is due to COSET processes; however, no clear modulations in the Fano factor, such as the ones predicted in Refs. [10,12] that discriminate between the mechanisms described above, have been observed so far. In this Letter, we present the first clear observation of such modulations.

We have investigated quantum dots formed in silicon nanowire metal-oxide-semiconductor field-effect transistors (MOSFETs), fabricated using a microelectronics technology based on $300 \mathrm{~mm}$ silicon-on-insulator (SOI) wafers. The low-temperature electronic conductance properties of such devices have been extensively studied in previous works [24-32], showing very robust Coulomb blockade characteristics. We performed conductance, current, and shot noise measurements in several small-size $(\approx 20 \times 30 \times 10 \mathrm{~nm}) \quad$-type devices using the setup described in Fig. 2, at a temperature of $0.3 \mathrm{~K}$ in a cryogen-free He-3 refrigerator. We measure the excess shot noise $S_{I I}$ in the quantum dot with a cross-correlation technique [33-35], where the current fluctuations on either side of the device are filtered at low temperature by $R L C$ tanks with a resonance frequency of approx. $3.5 \mathrm{MHz}$, and amplified using homemade ultralow noise preamplifiers. The cross-correlation of the outputs of both preamplifiers is then computed using a high speed digitizer. The dc and low frequency signals through the quantum dot (drain-source voltage $V_{\mathrm{DS}}$, dc current $I$, and differential conductance $\left.d I / d V_{\mathrm{DS}}\right)$ are applied and measured through the inductor of each $R L C$ tanks. This allows us to simultaneously measure $S_{I I}$ and $d I / d V_{\mathrm{DS}}$ as a function of $V_{\mathrm{DS}}$ and gate voltage $V_{g}$. In the most stable devices, we can compare the simultaneously acquired differential conductance and shot noise with the current, obtained in separate acquisitions, with

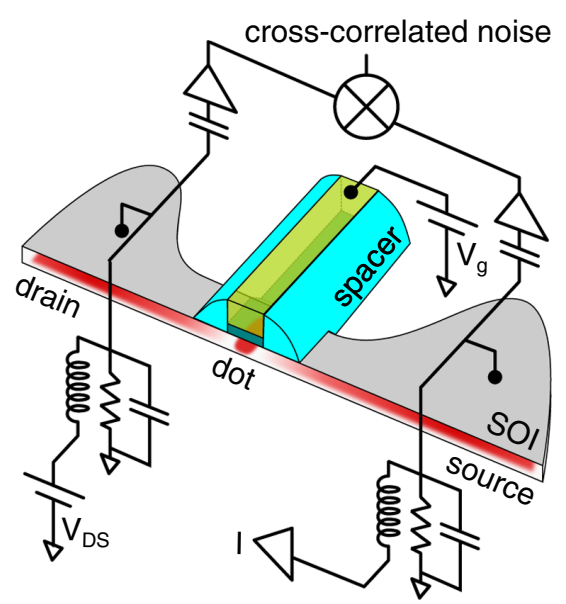

FIG. 2. Description of the experiment. The charge carriers in the silicon-on-insulator (SOI) channel are symbolized by the red clouds, showing the drain and source reservoirs on either side of the quantum dot. The spacers, realizing the tunnel barriers coupling the dot to the leads, are shown in light blue. The gate is shown in yellow and the gate insulator in dark gray. The electrical schematic is a simplified description of the measurement setup, including the pair of $R L C$ tanks used in the shot noise measurements.

very small drifts in gate voltage over extended periods of time (typically $2.5 \mathrm{mV}$ in a week). From the independents measurements of $S_{I I}$ and $I$, we compute an effective Fano factor $F=S_{I I} / 2 e I$.

Figure 3(a) shows measurements of $d I / d V_{\mathrm{DS}}$ in our best device, displaying two Coulomb diamonds (identified by the number of holes $N-1$ and $N$ ), analyzed in this Letter (measurements over the full range of $V_{g}$, as well as in other devices, are shown in the Supplemental Material [35]). The edges of the diamonds indicate the transitions involving ground states of the quantum dot, highlighted by the continuous yellow lines in Fig. 3(a). Similarly, the transitions involving excited states of the quantum dot, associated with resonances in $d I / d V_{\mathrm{DS}}$ outside of the diamonds, are highlighted by the dashed yellow lines. The negative slope resonance lines, corresponding to quantum dot transitions aligned with the electrochemical potential of the source, are constructed by comparing $d I / d V_{\mathrm{DS}}$ and $I\left(V_{\mathrm{DS}}\right)$ [35]. We extract the lever arms [35], as well as the charging and typical excited states energies $E_{c} \approx 8.7, \Delta^{*} \approx 2.2 \mathrm{meV}$. Note that these parameters are not constant over the full range of $V_{g}$, as the shape of the quantum dot is modified for large excursions in gate voltage [35]. $d I / d V_{\mathrm{DS}}$ is nonzero inside the diamonds, indicating the presence of cotunelling processes. The aforementioned condition $\Delta^{*}<E_{c} / 3$ to observe the various cotunneling regimes (1), (2), and (3) is fulfilled in our device. This is shown by extending the (yellow dashed) excited transitions lines into the Coulomb diamonds, yielding the light blue dashed lines in Fig. 3(a). As demonstrated below, these regimes indeed give rise to different shot noise contributions. 

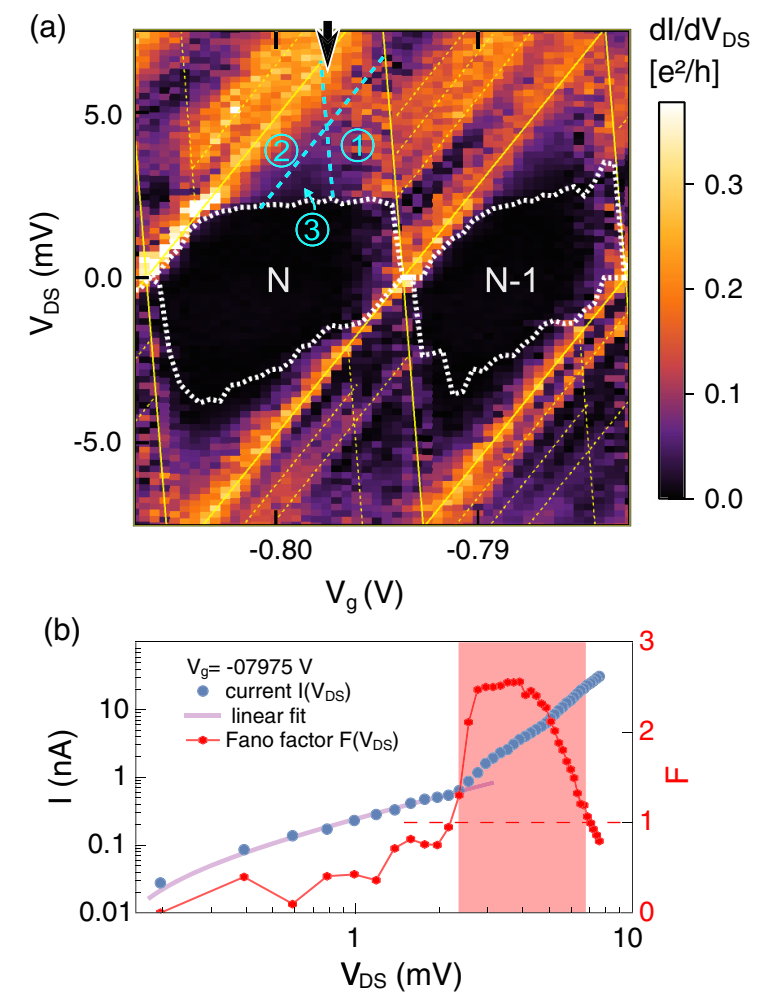

FIG. 3. (a) Differential conductance $d I / d V_{\text {DS }}$ measured as a function of gate voltage $V_{g}$ and drain-source voltage $V_{\mathrm{DS}}$. The continuous yellow lines locate the edges of the Coulomb diamonds, and the dashed yellow lines the excited states. The thick dotted white lines show the onset of inelastic cotunneling, extracted from current measurements (see below). The regions labeled (1), (2), and (3), separated by the light blue dashed lines (see text for their construction), correspond to the processes depicted in Fig. 1. (b) Current $I$ (blue symbols, left axis) and Fano factor $F$ (red symbols, right axis) as a function of $V_{\mathrm{DS}}$ in, respectively, log$\log$ and semi-log scale, for $V_{g}=-0.7975 \mathrm{~V}$ [black vertical arrow in (a) $]$. Continuous violet line: linear fit of $I\left(V_{\mathrm{DS}}\right)$ at low $V_{\mathrm{DS}}$ [35]. The horizontal red dashed line indicates the Poisson value $F=1$. The red area shows the inelastic cotunneling range, delimited by the value of $V_{\mathrm{DS}}$ at which $I\left(V_{\mathrm{DS}}\right)$ deviates from a linear behavior, and by the edge of the Coulomb diamond.

We quantitatively discriminate elastic and inelastic cotunneling regimes by exploiting our measurements of the current $I$ flowing across the dot. We check that the measured current [35] shows $V_{\mathrm{DS}}$ and $V_{g}$ dependences accurately matched by the resonance lines shown in Fig. 3(a). We then extract the onset of inelastic cotunneling, plotted as thick, dotted white lines in Fig. 3(a), by tracking, for a given value of $V_{g}$, at which $V_{\text {DS }}$ does $I$ change from a linear $V_{\text {DS }}$ dependence (characteristic of elastic cotunneling) to a power-law dependence (characterizing inelastic cotunneling) [36,37]. The onset closely follows the edges of the diamonds in vicinity of the charge degeneracy points, then clearly bifurcate inside the diamonds upon reaching the first excited state resonance. Furthermore, $d I / d V_{\mathrm{DS}}$ is clearly nonzero beyond the onset lines. Note that the onset lines do
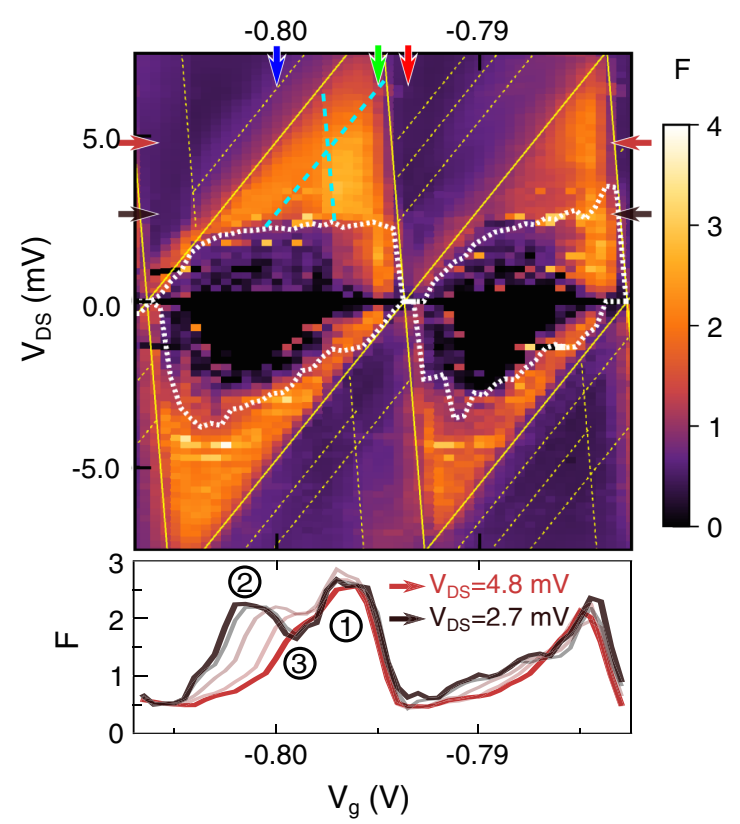

FIG. 4. Top: Fano factor $F$ measured as a function of $V_{g}$ and $V_{\text {DS }}$. The (full and dashed) yellow lines, the dashed blue lines, and the white dashed lines are the same as in Fig. 3. The blue, green, and red vertical arrows indicate the line cuts at constant $V_{g}$ shown in Fig. 5. The brown and dark brown horizontal arrows indicate the line cuts at constant $V_{\mathrm{DS}}$ shown in the bottom panel. Bottom: Line cuts of $F$ as a function of $V_{g}$, for $V_{\text {DS }}$ between 2.7 (dark brown) and $4.8 \mathrm{mV}$ (brown); the translucent lines correspond to intermediary values of $V_{\mathrm{DS}}$.

not correspond to constant $V_{\mathrm{DS}}$, which is reminiscent of energy renormalization due to inelastic cotunneling $[38,39]$. The extraction of the onset is illustrated in Fig. 3(b) for $V_{g}=-0.7975 \mathrm{~V}$, plotting $I\left(V_{\mathrm{DS}}\right)$ in log-log scale (left $Y$ axis, blue dots). Below $V_{\mathrm{DS}} \approx 2.3 \mathrm{mV}$, defining the onset, $I\left(V_{\mathrm{DS}}\right)$ is linear (the violet line is a linear fit of the data in that range), then takes a power law $\left(V_{\mathrm{DS}}\right)^{\alpha}$, with $\alpha \approx 3-7$ (red area) [35]. Plotting the Fano factor $F$ on the same graph (right $Y$ axis, red symbols) shows the noise contribution of each cotunneling regime: while $F$ stays below the Poissonian value $F=1$ in the elastic cotunneling regime, it rapidly shoots up to significantly large values $F \approx 2.5$ in the inelastic cotunneling regime, then decreases back to sub-Poissonian values outside of the Coulomb diamond.

Figure 4 shows a map of $F$ over the same range of $V_{\mathrm{DS}}$ and $V_{g}$ as in Fig. 3(a), also including the resonance lines and the inelastic cotunneling onset presented in the latter. With a few notable exceptions, which we discuss below, $F$ is, very clearly, only above the Poisson value in the regime of inelastic cotunneling. In the elastic cotunneling regime, $F$ generally takes values smaller than 1 . Note that given the small magnitude of both $I$ and $S_{I I}$ in this regime, $F$ presents large relative fluctuations. For clarity, we have set $F=0$ whenever either $I<50 \mathrm{pA}$ or $S_{I I}<2 \times 10^{-29} \mathrm{~A}^{2} / \mathrm{Hz}$. Outside of the diamonds, $F$ is close to 0.5 , the value 
for sequential tunneling across a dot with symmetric barriers [1].

We now discuss the variations of $F$ in regions (1), (2), and (3) in the inelastic cotunneling regime. As shown in Fig. 4, F presents sizable modulations, on the order of unity, depending on the position of the excited states transitions with respect to source and drain electrochemical potentials, that match the regions delimited by the blue dashed lines. This appears clearly when taking line cuts of the data at fixed $V_{\mathrm{DS}}$, as a function of $V_{g}$ : just above the inelastic cotunneling onset, at $V_{\mathrm{DS}}=2.7 \mathrm{mV}$ (dark brown line in the bottom panel of Fig. 4), $F$ is nonmonotonic inside the diamond, with a local minimum at $F \approx 1.7$ in the region labeled (3) in Fig. 3. Larger values of $F$ on either side of that minimum can thus be attributed to COSET processes such as (1) and (2). As $V_{\mathrm{DS}}$ is increased, all cotunneling processes end with an excited transition in the transport window, and the local minimum vanishes, as illustrated by the line cut at $V_{\mathrm{DS}}=$ $4.8 \mathrm{mV}$ (brown line in the bottom panel of Fig. 4). The measured modulations of $F$ in the inelastic cotunneling regime mimic those predicted in Refs. [10,12], indicating that super-Poissonian current fluctuations indeed stem from different mechanisms depending on the position of the excited states transitions with respect to drain and source electrochemical potentials.

Figure 4 shows that, unexpectedly, $F$ takes superPoissonian values even for $V_{\mathrm{DS}}$ below the inelastic cotunneling onset, close to the edges of the diamonds. This regime of comparatively large fluctuations is quantitatively different from the inelastic cotunneling regime, as illustrated by Fig. 5, where we have plotted the $V_{\text {DS }}$ dependence of both $I$ and $F$, in, respectively, $\log$-log and semi-log scale, for three values of $V_{g}$ leading to different transport regimes. These values are indicated by vertical arrows in Fig. 4: for $V_{g}=-0.7935 \mathrm{~V}$ (red), Coulomb blockade is lifted, and the tunneling through the quantum dot is sequential, leading to comparatively large currents. This regime yields usual, subPoissonian values of $F$, saturating at the symmetric value $F=0.5$ at large $V_{\mathrm{DS}}$. The data at $V_{g}=-0.8 \mathrm{~V}$ (blue) are similar to the ones shown in Fig. 3(b), first showing a linear $I\left(V_{\mathrm{DS}}\right)$ behavior in the elastic cotuneling regime (blue line), followed by an approximative $\left(V_{\mathrm{DS}}\right)^{4}$ dependence in the inelastic cotunneling regime. As discussed above, this leads to super-Poissonian values of $F$ only in the inelastic cotunneling regime. The data at $V_{g}=-0.795 \mathrm{~V}$ (green), close to the edge of the diamond, differ markedly from the previous regimes: indeed, while here $I\left(V_{\mathrm{DS}}\right)$ remains essentially linear, taking intermediary values in the $\mathrm{nA}$ range, $F$ takes large, super-Poissonian values in the whole range of $V_{\mathrm{DS}}$, in particular, well below the inelastic cotunneling onset (appearing as the edge of the red shaded areas in both panels of Fig. 5). This behavior of comparatively large, linear $I\left(V_{\mathrm{DS}}\right)$ together with super-Poissonian fluctuations below the inelastic cotunneling onset appears in both $N-1$ and $N$ diamonds shown in Fig. 4, for positive

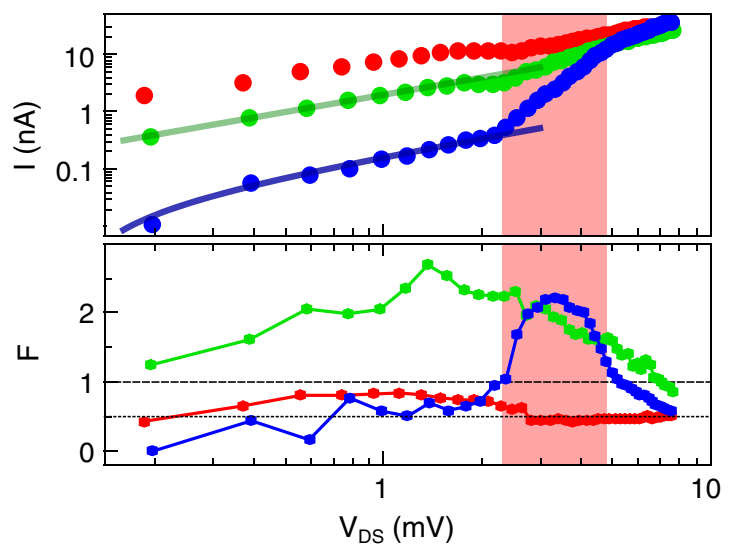

FIG. 5. Current $I$ (top panel) and Fano factor $F$ (bottom panel) as a function of $V_{\mathrm{DS}}$ in, respectively, log-log and semi-log scale, for three different values of $V_{g}$, located by the vertical arrows in Fig. 4: $V_{g}=-0.7935 \mathrm{~V}$ (red), $V_{g}=-0.795 \mathrm{~V}$ (green), and $V_{g}=-0.8 \mathrm{~V}$ (blue). Continuous lines in the top panel: linear fits of the low- $V_{\mathrm{DS}}$ data. The sequential tunneling $(F=0.5)$ and Poissonian $(F=1)$ values of $F$ are indicated by, respectively, horizontal dotted and dashed lines in the bottom panel. In both panels, the red area indicates the inelastic cotunneling range defined for $V_{g}=-0.8 \mathrm{~V}$.

and negative $V_{\mathrm{DS}}$ (see also Ref. [35] for maps of the measured current versus $V_{\mathrm{DS}}$ and $V_{g}$ ). Note, however, that (i) it is not observed in all Coulomb diamonds for this device, and (ii) surprisingly, it only appears on one side of each diamond, namely, towards the $N \leftrightarrow N-1$ degeneracy point of the $N$-hole diamond. This asymmetry is also seen to some extent in the inelastic cotunneling regime [35]. To our knowledge, there is no straightforward mechanism leading to enhanced current and fluctuations in the elastic cotunneling regime. Among the possible explanations, dynamical channel blockade [6] can be ruled out by the fact that it occurs outside of the Coulomb diamonds, and that it requires the presence of excited states at energies below $2 \mathrm{meV}$, which are not clearly observed in the differential conductance. Another mechanism stems from the possible presence of nearby charge traps poorly coupled to the leads. Single dopants located below the spacers can act as such charge traps [27], randomly switching the conduction state of the quantum dot, thereby increasing current fluctuations. Previous studies on similar nanowire MOSFETs showed that the presence of such traps is characterized in $d I / d V_{\mathrm{DS}}$ measurements by large scale $V_{g}$ periodicity and phase shifts in the Coulomb diamonds [27,40-42]. While these features do not appear clearly in the measured conductance, similar ones can be seen in the measured shot noise [35]; it is thus not entirely unlikely that the presence of one or more charge traps gives rise to the measured enhanced fluctuations at low $V_{\mathrm{DS}}$.

In summary, we have observed for the first time clear modulations of super-Poissonian fluctuations in the inelastic cotunneling regime, which stem from distinct 
mechanisms depending on the chemical potential of the quantum dot. We have also observed previously unreported enhanced fluctuations in the elastic cotunneling regime, which might be attributed to nearby charge traps.

We warmly thank J. Splettstoesser, M. Misiorny, C. Flindt, Ch. Strunk, A. Donarini, R. Maurand, F. Portier, and C. Altimiras for enlightening discussions. This work was funded by the CEA (Programme Transversal Nanosciences 15.1.4c-NANOSiN) and the ERC (ERC-2015-STG COHEGRAPH). M. Seo acknowledges support from the Enhanced Eurotalents programme.

*Corresponding author. francois.parmentier@cea.fr

[1] Y. Blanter and M. Büttiker, Phys. Rep. 336, 1 (2000).

[2] M. Reznikov, M. Heiblum, H. Shtrikman, and D. Mahalu, Phys. Rev. Lett. 75, 3340 (1995).

[3] A. Kumar, L. Saminadayar, D. C. Glattli, Y. Jin, and B. Etienne, Phys. Rev. Lett. 76, 2778 (1996).

[4] H. Birk, M. J. M. de Jong, and C. Schönenberger, Phys. Rev. Lett. 75, 1610 (1995).

[5] E. V. Sukhorukov, G. Burkard, and D. Loss, Phys. Rev. B 63, 125315 (2001).

[6] W. Belzig, Phys. Rev. B 71, 161301 (2005).

[7] A. Thielmann, M. H. Hettler, J. König, and G. Schön, Phys. Rev. Lett. 95, 146806 (2005).

[8] E. Onac, F. Balestro, B. Trauzettel, C. F. J. Lodewijk, and L. P. Kouwenhoven, Phys. Rev. Lett. 96, 026803 (2006).

[9] I. Weymann, J. Barnaś, and S. Krompiewski, Phys. Rev. B 76, 155408 (2007).

[10] J. Aghassi, M. H. Hettler, and G. Schön, Appl. Phys. Lett. 92, 202101 (2008).

[11] A. Carmi and Y. Oreg, Phys. Rev. B 85, 045325 (2012).

[12] K. Kaasbjerg and W. Belzig, Phys. Rev. B 91, 235413 (2015).

[13] Y. Zhang, L. DiCarlo, D. T. McClure, M. Yamamoto, S. Tarucha, C. M. Marcus, M. P. Hanson, and A. C. Gossard, Phys. Rev. Lett. 99, 036603 (2007).

[14] O. Zarchin, Y. C. Chung, M. Heiblum, D. Rohrlich, and V. Umansky, Phys. Rev. Lett. 98, 066801 (2007).

[15] C. Fricke, F. Hohls, W. Wegscheider, and R. J. Haug, Phys. Rev. B 76, 155307 (2007).

[16] Y. Okazaki, S. Sasaki, and K. Muraki, Phys. Rev. B 87, 041302 (2013).

[17] M.-C. Harabula, V. Ranjan, R. Haller, G. Fülöp, and C. Schönenberger, Phys. Rev. B 97, 115403 (2018).

[18] S. De Franceschi, S. Sasaki, J. M. Elzerman, W. G. van der Wiel, S. Tarucha, and L. P. Kouwenhoven, Phys. Rev. Lett. 86, 878 (2001).

[19] M. R. Wegewijs and Y. V. Nazarov, arXiv:cond-mat/ 0103579 .

[20] R. Schleser, T. Ihn, E. Ruh, K. Ensslin, M. Tews, D. Pfannkuche, D. C. Driscoll, and A. C. Gossard, Phys. Rev. Lett. 94, 206805 (2005).
[21] V. N. Golovach and D. Loss, Phys. Rev. B 69, 245327 (2004).

[22] S. Andergassen, V. Meden, H. Schoeller, J. Splettstoesser, and M. R. Wegewijs, Nanotechnology 21, 272001 (2010).

[23] R. Gaudenzi, M. Misiorny, E. Burzurí, M. R. Wegewijs, and H. S. J. van der Zant, J. Chem. Phys. 146, 092330 (2017).

[24] M. Sanquer, M. Specht, L. Ghenim, S. Deleonibus, and G. Guegan, Phys. Rev. B 61, 7249 (2000).

[25] M. Boehm, M. Hofheinz, X. Jehl, M. Sanquer, M. Vinet, B. Previtali, D. Fraboulet, D. Mariolle, and S. Deleonibus, Phys. Rev. B 71, 033305 (2005).

[26] M. Hofheinz, X. Jehl, M. Sanquer, G. Molas, M. Vinet, and S. Deleonibus, Appl. Phys. Lett. 89, 143504 (2006).

[27] M. Hofheinz, X. Jehl, M. Sanquer, G. Molas, M. Vinet, and S. Deleonibus, Eur. Phys. J. B 54, 299 (2006).

[28] M. Pierre, R. Wacquez, X. Jehl, M. Sanquer, M. Vinet, and O. Cueto, Nat. Nanotechnol. 5, 133 (2010).

[29] E. Prati, M. De Michielis, M. Belli, S. Cocco, M. Fanciulli, D. Kotekar-Patil, M. Ruoff, D. P. Kern, D. A. Wharam, J. Verduijn, G. C. Tettamanzi, S. Rogge, B. Roche, R. Wacquez, X. Jehl, M. Vinet, and M. Sanquer, Nanotechnology 23, 215204 (2012).

[30] B. Roche, E. Dupont-Ferrier, B. Voisin, M. Cobian, X. Jehl, R. Wacquez, M. Vinet, Y.-M. Niquet, and M. Sanquer, Phys. Rev. Lett. 108, 206812 (2012).

[31] R. Lavieville, F. Triozon, S. Barraud, A. Corna, X. Jehl, M. Sanquer, J. Li, A. Abisset, I. Duchemin, and Y.-M. Niquet, Nano Lett. 15, 2958 (2015).

[32] B. Voisin, R. Maurand, S. Barraud, M. Vinet, X. Jehl, M. Sanquer, J. Renard, and S. De Franceschi, Nano Lett. 16, 88 (2016).

[33] L. DiCarlo, Y. Zhang, D. T. McClure, C. M. Marcus, L. N. Pfeiffer, and K. W. West, Rev. Sci. Instrum. 77, 073906 (2006).

[34] F. D. Parmentier, L. N. Serkovic-Loli, P. Roulleau, and D. C. Glattli, Phys. Rev. Lett. 116, 227401 (2016).

[35] See Supplemental Material at http://link.aps.org/ supplemental/10.1103/PhysRevLett.121.027701 for a description of the experimental setup, as well as additional data and analysis.

[36] D. V. Averin and Y. V. Nazarov, Phys. Rev. Lett. 65, 2446 (1990).

[37] D. C. Glattli, C. Pasquier, U. Meirav, F. I. B. Williams, Y. Jin, and B. Etienne, Z. Phys. B 85, 375 (1991).

[38] J. V. Holm, H. I. Jørgensen, K. Grove-Rasmussen, J. Paaske, K. Flensberg, and P. E. Lindelof, Phys. Rev. B 77, 161406 (2008).

[39] J. Splettstoesser, M. Governale, and J. König, Phys. Rev. B 86, 035432 (2012).

[40] C. C. Escott, F. A. Zwanenburg, and A. Morello, Nanotechnology 21, 274018 (2010).

[41] V. N. Golovach, X. Jehl, M. Houzet, M. Pierre, B. Roche, M. Sanquer, and L. I. Glazman, Phys. Rev. B 83, 075401 (2011).

[42] B. J. Villis, A. O. Orlov, S. Barraud, M. Vinet, M. Sanquer, P. Fay, G. Snider, and X. Jehl, Appl. Phys. Lett. 104, 233503 (2014). 